\title{
Mathematical Modeling of Charge Transfer Processes in Polymeric lonites During the Water Vapor Sorption
}

\author{
Igor Ivanov ${ }^{1, *}$ and Oksana Ivanova ${ }^{2}$ \\ ${ }^{1}$ Russian State Agricultural University, Kaluga branch, RU-248007, Kaluga, Russia \\ ${ }^{2}$ Moscow State Technological University "STANKIN", RU-127055, Moscow, Russia
}

\begin{abstract}
Based on a thermodynamic approach, a mathematical model of charge transfer processes in the polymeric ionites (ion-exchange materials) during the water vapor sorption was developed. Theoretical dependences of the resistance of a polymeric ionite on the relative air humidity over the range $10-100 \%$ at physically reasonable values of the parameters of the system were obtained. The comparison of theoretical and experimental dependences has been performed. Possible reasons for and criteria of the phase transitions that lead to stepwise change of ionite resistance were discussed. The developed mathematical model adequately describes the available experimental data and allows formulating practical recommendations on selecting optimal polymeric coatings for the electrode units of molecular-electronic humidity gauges.
\end{abstract}

\section{Introduction}

Measuring the humidity of air over a wide range at various temperatures is a long-standing problem, which is still very important. Today, humidity gauges are successfully elaborated using molecular electronic technologies. In comparison with hygrometers, devices unitizing humidity-induced changes in elasticity, the precision of the new gauges is much higher, with the covered humidity range being virtually unlimited. These gauges are used at different facilities with requirements to maintain humidity in an certain range such as laboratories, pharmaceutical companies, poultry farms, hothouses, warehouses, drying-rooms, museums, and others.

The sensor in the new gauges is typically a polymeric ionite (PI) placed between two electrodes. The absorption of water by such a polymer is accompanied by dissociation of its surface ionogenic groups (IGs), which, in turn, speeds up the formation of free charge carriers and, hence, increases the conductance of the polymer. From a practical point of view, such a polymer must have a single-valued dependence of the conductance (or resistance) on the humidity and temperature of the medium.

The mechanism of the conduction of PIs, especially at low humidity, is still poorly understood because; in most cases , the conductance of a polymer at equilibrium with a solution $[1,2]$ was calculated, while the equilibrium with not water vapors was not considered.

The development of model of the interaction of PIs is quite difficult due the absence of information on the effect of long-range electrostatic forces on the characteristics of sorption. For example, the thermodynamic approach developed in [3] was limited to describing the Coulomb interaction without taking into account the effect of screening of the charges and the influence of the electric double layer. The predicted dependences of the conduction on the humidity deviated markedly from the experimental results, especially at low humidity. Indeed, since an adsorbed film is, in fact, a single-component solution with a characteristic screening distance, even a small change in the thickness of the film may lead to a significant distortion of the screening cloud and, hence, to changes in the electrostatic energy of ions and the conductance of the entire system. To correct the aforementioned shortcomings, in work [4] the mathematical model, which takes into account the effects of the surface charge and the Debye screening in the films, has been developed.

However, for a more detailed description of charge transfer processes in porous PIs and comparison the theoretical results with the experimental data, the iondipole interaction during the sorption of polarized molecules should be included in the model. Moreover, the possible reasons and criteria of the phase transitions, which lead to a jumping change of ionite resistance, should be defined more precisely and connected with the relevant parameters of real physical systems, because such phase transitions into PI sample during the water vapor sorption induce the nonstability in hygrometer readings. The development of such mathematical model (based on thermodynamic approach with the exact calculation of the electrostatic energy of ions) is the main goal of the present work.

\section{Theoretical Analysis}

Let us first give a brief description of the structure of PIs. The surface of PI is composed of crosslinked 
organic polymer chains (a kind of matrix) with attached inorganic residues (ion-exchange or ionogenic groups) capable of dissociating in water solutions.

In resent years, a model postulating the formation of two phases of ionite (composed of organic chains hydrophobic phase, and IGs with sorbed water molecules hydrophilic phase) has gained popularity [3, 7-9]. According to this model, the separation of the phases is accompanied by the formation of clusters composed of IGs, ions, and water molecules inside the organic polymer matrix. As a result, pores with flexible walls are formed. When the humidity tends to zero, such pores disappear; therefore, it is more correct to call them quasi-pores [7]. Pores are connected via channels (quasi-channels), which arise in areas where the density of polymer chains is low. The size of quasi-pores ranges from 2 to $100 \mathrm{~nm}$, while the width of channels is about $1 \mathrm{~nm}$ [7]. Electron microscope analysis confirmed the existence of a large number of pores and channels in PIs [8, 10].

The sorption of moisture from the environment is accompanied by the dissociation of IGs, a process that makes the system conductive, with electric current flowing through circuits formed by channels and hydrophilic clusters. To describe the conduction in PIs, which are nonuniform media, we used the concepts underlying the theory of percolation [11]. According to [11], the current flowing through a nonuniform medium is determined by the lowest conductivity areas on the route between the electrodes of the measuring unit. Thus, it is sufficient to calculate the conductivity of these areas.

Let us consider an area of an ionite containing the critical number $N$ of IGs bonded to the polymer matrix (polymer chain). Let the humidity be such that the formation of the primary hydration shells of the IGs (for most of the ionites, this situation corresponds to a range of relative humidity $P / P_{S}$ from $\sim 0,01$ to 0,1$)$. Then, the subsequent sorption occurs due to the interaction of newly arriving water molecules with hydration shells and the osmotic effect. The total thermodynamic potential of the system can be presented as

$$
\Phi=\Phi_{0}+\Phi_{W}+\Phi_{I}+\Phi_{P M} .
$$

Here, $\Phi_{0}$ is the thermodynamic potential of the ionite in the standard state, which was defined as the state in which the ionite contains only the water entering into the hydration shells ( $N_{W 0}$ molecules), and $\Phi_{W}$ is the change in the thermodynamic potential caused by the subsequent condensation of water. Assuming that the structure of the water not entering into the hydration shells of the IGs is identical to that of bulk liquid water, we obtained

$$
\Phi_{W}=-\left(N_{W}-N_{W 0}\right) T \ln \left(P / P_{S}\right),
$$

where $N_{W}-N_{W 0}$ is the number of water molecules not entering into the hydration shells of the IGs, $N_{W}$ is the total number of water molecules in the system, $P / P_{S}$ is the relative air humidity, $P_{S}$ is the saturation vapor pressure, and $T$ is the absolute temperature expressed in energy units.

The potential $\Phi_{I}$ takes into account the contributions from the ionic subsystem, more specifically, those associated with the dissociation of IGs, the formation of their hydration shells, and the formation of a solution composed of a hydrated ions and free water, as well as the entropy contribution associated with the different configurations in the positioning of dissociated and nondissociated IGs in the polymer matrix. For simplicity, we assumed that the IGs are composed of cations and anions of the same valence. Then,

$$
\begin{gathered}
\Phi_{I}=\alpha N E_{D}+T \alpha N \ln \frac{\alpha}{1-\alpha} \\
+T N \ln \frac{N(1-\alpha)}{N+N_{W}-N_{W 0}-\alpha(n-1) N} \\
+T \alpha N \ln \frac{\alpha N}{N+N_{W}-N_{W 0}-\alpha(n-1) N} \\
+T\left(N_{W}-N_{W 0}-\alpha n N\right) \ln \frac{N_{W}-N_{W 0}-\alpha n N}{N+N_{W}-N_{W 0}-\alpha(n-1) N},
\end{gathered}
$$

where $\alpha$ is the degree of dissociation of IGs; $n$ is the conventional number of hydration of ions in a singlecomponent solution [7]; $E_{D}=E_{0}+E_{E L}$ is the dissociation energy, which consists of the energy of abstraction of the ion from the polymer chain $\left(E_{0}\right)$ and the change in its electrostatic energy $\left(E_{E L}\right) ; N_{W}-N_{W 0}-$ $\alpha n N$ is the number of absorbed free water molecules. The last three summands were obtained (by using the Sterling formula) from the expression for total entropy of system of particles:

$$
S=\ln \left[\frac{N !}{(\alpha N) !(N-\alpha N) !} \frac{\left(N+N_{W}-N_{W 0}-\alpha n N+\alpha N\right) !}{N !(\alpha N) !\left(N_{W}-N_{W 0}-\alpha n N\right) !}\right] .
$$

The total entropy consists of the entropy of positioning of dissociated and nondissociated groups (the first multiplier) and the entropy of mixing of particles of all sorts (the second multiplier).

The last term in (1) describes the change in the state of the polymer matrix during swelling and can be considered as the energy of elastic deformation of the polymer chains. The dependence of the elastic deformation energy on the humidity can be obtained in a similar way [2]

$$
\Phi_{P M}=3 T\left(\frac{V_{W}}{V_{P}}\right)^{2} \frac{N_{W}^{2}}{N},
$$

where $V_{W}$ is the molecular volume of water and $V_{P}$ is the polymer chain volume per IG. Expression (4) can be obtained from Hooke's law with an elasticity modulus of $3 T / V_{P}$.

Finally, denoting $\beta=N_{W} / N$ (relative moisture content), $\beta_{0}=N_{W 0} / N$ is the conventional number of hydration of IGs, and $U=6\left(V_{W} / V_{P}\right)^{2}$, we presented the total thermodynamic potential of the system (1) per number of IGs $N(\phi=\Phi / N)$ as

$$
\begin{array}{r}
\phi=-T\left(\beta-\beta_{0}\right) \ln \left(P / P_{S}\right)+\frac{1}{2} U T \beta^{2}+\alpha E_{D} \\
+T \alpha \ln \frac{\alpha}{1-\alpha}+T \ln \frac{1-\alpha}{1+\beta-\beta_{0}-\alpha(n-1)} \\
+T \alpha \ln \frac{\alpha}{1+\beta-\beta_{0}-\alpha(n-1)} \\
+T\left(\beta-\beta_{0}-\alpha n\right) \ln \frac{\beta-\beta_{0}-\alpha n}{1+\beta-\beta_{0}-\alpha(n-1)} .
\end{array}
$$


At equilibrium, the thermodynamic potential $\phi$ takes its minimum value: $\partial \phi / \partial \alpha=0, \partial \phi / \partial \beta=0$. As a result, the expressions for the relative moisture content $\beta$ and the degree of dissociation of IGs $\alpha$ read

$$
\left\{\begin{array}{l}
P / P_{S}=\frac{\beta-\beta_{0}-\alpha n}{1+\beta-\beta_{0}-\alpha(n-1)} \exp \left(\frac{\alpha}{T} \frac{\partial E_{D}}{\partial \beta}+U \beta\right), \\
\frac{\alpha^{2}}{1-\alpha} \frac{\left(1+\beta-\beta_{0}-\alpha(n-1)\right)^{n-1}}{\left(\beta-\beta_{0}-\alpha n\right)^{n}}=\exp \left(-\frac{1}{T} \frac{\partial\left(\alpha E_{D}\right)}{\partial \alpha}\right) .
\end{array}\right.
$$

At large values of the relative moisture content, $\partial\left(\alpha E_{D}\right) / \partial \alpha \rightarrow E_{0}, \partial E_{D} / \partial \beta \rightarrow 0$, and, therefore, formula (6) transforms into the standard expression for the concentration dependence of the vapor pressure over solution [12], while formula (7) takes the form of the law of mass action [13]. Formulas (6) and (7) make it possible not only to determine the values of $\alpha$ and $P / P_{S}$ for the bulk phase, but also to examine how they change in the case of sorbed thin water films.

The dependence of the energy $E_{D}$, more specifically, of its electrostatic component $E_{E L}$, on the degree of dissociation and the water content is determined by the interaction of the charged species with the ionic cloud and with the boundaries of the areas occupied by the solvent. To calculate $E_{E L}$, it is necessary to know the distribution of the potential of a point charge $q$ in a solid dielectric - liquid - solid dielectric three-layer structure. Let the solution from a plane thin (compared to the characteristic size of the hydrophilic area) film of thickness $L$ at the surface of an ionite, and let the dielectric permittivity of the solution and the environment be $\varepsilon$ and $\varepsilon_{1}$, respectively.

We used the method described in [14] but additionally took into account the charge of one of the surfaces and the Debye screening in a single-component solution. In this case, the point charge potential distribution in the liquid layer (the Poisson - Boltzmann equation) and in the two solid dielectric layers (the Laplace equation) reads

$$
\Delta \varphi_{0}=\kappa^{2} \varphi_{0}-\frac{4 \pi q}{\varepsilon} \delta\left(\mathbf{r}-\mathbf{r}_{0}\right) ; \quad \Delta \varphi_{1,2}=0,
$$

where $\kappa=\left[4 \pi q^{2} c /(\varepsilon T)\right]^{1 / 2}$ is the reciprocal Debye screening distance of a single-component solution of concentration $c$ and $\mathbf{r}_{0}$ is the radius-vector of the given ion.

Assuming that the dimensions of the layers in the $X$ and $Y$ directions are much larger then their dimensions in the $Z$ direction (thickness) and Debye screening distance, we performed the two-dimentional Fouriertransformation of this equation for the three layers to obtain

$$
\begin{aligned}
& \frac{\partial^{2} \varphi_{0}(\mathbf{k}, z)}{\partial z^{2}}-k_{0}^{2} \varphi_{0}(\mathbf{k}, z)=\frac{4 \pi q}{\varepsilon} \delta\left(z-z_{0}\right), 0<z<L \\
& \frac{\partial^{2} \varphi_{1}(\mathbf{k}, z)}{\partial z^{2}}-k^{2} \varphi_{1}(\mathbf{k}, z)=0, \quad z>L \\
& \frac{\partial^{2} \varphi_{2}(\mathbf{k}, z)}{\partial z^{2}}-k^{2} \varphi_{2}(\mathbf{k}, z)=0, \quad z<0
\end{aligned}
$$

where $k_{0}^{2}=k^{2}+\kappa^{2}$.
The boundary conditions for system (8) are the equality of the potential at either boundary and the continuity of the normal component of the electric displacement at the upper boundary $(z=L)$; at the lower boundary $(z=0)$, it experiences a jump of $4 \pi \sigma$, where $\sigma$ is the surface charge density.

Then, for the Fourier-component of the potential at $z \rightarrow z_{0}$, we can write

$$
\begin{gathered}
\varphi_{0}\left(\mathbf{k}, z_{0}\right)=\frac{2 \pi q}{\varepsilon k_{0}} \sum_{m=1}^{\infty}\left[f^{2 m-1} \exp \left(-2 k_{0}\left(L(m-1)+z_{0}\right)\right)\right. \\
\left.+2 f^{2 m} \exp \left(-2 k_{0} L m\right)+f^{2 m-1} \exp \left(-2 k_{0}\left(L m-z_{0}\right)\right)\right] \\
+\frac{2 \pi q}{\varepsilon k_{0}} \exp \left(-k_{0}\left|z-z_{0}\right|\right) \\
+\frac{4 \pi \sigma}{\varepsilon k_{0}+\varepsilon_{1} k}(2 \pi)^{2} \delta(\mathbf{k}) \frac{\exp \left(-k_{0} z_{0}\right)+f \exp \left(-k_{0}\left(2 L-z_{0}\right)\right)}{1-f^{2} \exp \left(-2 k_{0} L\right)} .
\end{gathered}
$$

The function $f(k)=\left(\varepsilon k_{0}-\varepsilon_{1} k\right) /\left(\varepsilon k_{0}+\varepsilon_{1} k\right)$ only slightly changes over the range $0<k<\infty$, so that it can be set constant, $f=\left(\varepsilon-\varepsilon_{1}\right) /\left(\varepsilon+\varepsilon_{1}\right)$. This assumption is physically reasonable, since $\varepsilon_{1}<<\varepsilon$.

To avoid a cumbersome (and hardly justified) averaging over various spatial configurations of the ions, we postulated that the charge is uniformly distributed over the plane passing through the middle of the film $\left(z_{0}=L / 2\right)$. Then, after performing the inverse Fouriertransformation, excluding the self-action of the charge, and taking into account that $E_{E L}=q \varphi_{0}$, we obtained

$$
E_{E L}=-\frac{q^{2} \kappa}{\varepsilon}-\frac{2 q^{2}}{\varepsilon L} \ln \left(1-f e^{-\kappa L}\right)+\frac{4 \pi \sigma q}{\varepsilon \kappa} \frac{e^{-\kappa L / 2}}{1-e^{-\kappa L}} .
$$

The terms in (9) correspond to the contributions to the electrostatic energy from the bulk of the screening cloud, the charges induced in the solid dielectric layers due to the finite thickness of the liquid layer, and the charge of the surface, respectively. In the absence of the surface charge $(\sigma=0)$ and Debye screening $(\kappa \rightarrow 0)$ formula (9) transforms into the expression derived in [4, 14].

Some of the parameters in (9) can be expressed through $\alpha$ and $\beta: L=\beta V_{W} / s$, where $s$ is the surface area per IG, $\sigma=-\alpha q / s$ (it was assumed that the charges of one sign are located in the solution, while the charges of opposite sign are located at the surface, so that the ionite phase as a whole remains electrically neutral), and $\kappa=\left[\left(4 \pi q^{2} /\left(\varepsilon T V_{W}\right)\right)(\alpha / \beta)\right]^{1 / 2}$. Then, the dissociation energy can be presented as

$$
\begin{aligned}
E_{D}=E_{0}- & \frac{C T}{\beta}\left(\frac{\xi \sqrt{\alpha \beta}}{2}+\ln (1-f \exp (-\xi \sqrt{\alpha \beta}))\right) \\
& -\frac{\xi T \sqrt{\alpha \beta} \exp (-\xi \sqrt{\alpha \beta} / 2)}{1-\exp (-\xi \sqrt{\alpha \beta})},
\end{aligned}
$$

where $C=2 q^{2} s /\left(\varepsilon T V_{W}\right)$ and $\xi=\left[4 \pi q^{2} V_{W} /\left(\varepsilon T s^{2}\right)\right]^{1 / 2}$ are dimensionless quantities.

The main contribution in ion-dipole interaction belongs to water molecules in hydration shells of ions in the solution. Therefore, the ion-dipole interaction energy takes the form

$$
E_{i d}=-n \frac{q p}{\varepsilon r^{2}}
$$


where $p$ is the dipole moment of water molecule and $r$ is the characteristic ion-to-dipole distance.

Considered, that $p=\delta q r$ and $r=\left[3 V_{W} /(4 \pi)\right]^{1 / 3}$, where $\delta q$ is effective charge of dipole edges $(\delta \sim 0,1)$, we can write (10) through dimensionless quantities

$$
E_{i d}=-\frac{n \delta}{12^{1 / 3}}(C \xi)^{2 / 3} T .
$$

Finally, the dissociation energy takes the form

$$
\begin{gathered}
E_{D}=E_{0}-\frac{C T}{\beta}\left(\frac{\xi \sqrt{\alpha \beta}}{2}+\ln (1-f \exp (-\xi \sqrt{\alpha \beta}))\right) \\
-\frac{\xi T \sqrt{\alpha \beta} \exp (-\xi \sqrt{\alpha \beta} / 2)}{1-\exp (-\xi \sqrt{\alpha \beta})}-\frac{n \delta}{12^{1 / 3}}(C \xi)^{2 / 3} T .
\end{gathered}
$$

This formula is valid at all physically reasonable values of system parameters (water content and degree of dissociation), even at lowest humidity, when hydration shells of ions are not formed completely (the dependence $n(\beta)$ is need). For this reason, formula (11) can be used over the full relative humidity range.

Now, we can write the derivatives entering into the system of equations (6), (7)

$$
\begin{aligned}
& \frac{\partial E_{D}}{\partial \beta}=\frac{C T}{\beta^{2}}\left(\frac{\xi \sqrt{\alpha \beta}}{4} \frac{1-3 f \exp (-\xi \sqrt{\alpha \beta})}{1-f \exp (-\xi \sqrt{\alpha \beta})}+\ln (1-f \exp (-\xi \sqrt{\alpha \beta}))\right) \\
& -\frac{T \xi \sqrt{\alpha \beta} \exp (-\xi \sqrt{\alpha \beta} / 2)}{2 \beta(1-\exp (-\xi \sqrt{\alpha \beta}))^{2}}\left(1-\frac{\xi \sqrt{\alpha \beta}}{2}-\exp (-\xi \sqrt{\alpha \beta})\left(1+\frac{\xi \sqrt{\alpha \beta}}{2}\right)\right) ; \\
& \frac{\partial\left(\alpha E_{D}\right)}{\partial \alpha}=E_{0}-\frac{n \delta}{12^{1 / 3}}(C \xi)^{2 / 3} T \\
& -\frac{C T}{\beta}\left(\frac{\xi \sqrt{\alpha \beta}}{4} \frac{3-f \exp (-\xi \sqrt{\alpha \beta})}{1-f \exp (-\xi \sqrt{\alpha \beta})}+\ln (1-f \exp (-\xi \sqrt{\alpha \beta}))\right) \\
& -\frac{T \xi \sqrt{\alpha \beta} \exp (-\xi \sqrt{\alpha \beta} / 2)}{2(1-\exp (-\xi \sqrt{\alpha \beta}))^{2}}\left(3-\frac{\xi \sqrt{\alpha \beta}}{2}-\exp (-\xi \sqrt{\alpha \beta})\left(3+\frac{\xi \sqrt{\alpha \beta}}{2}\right)\right) .
\end{aligned}
$$

\section{Results and Discussion}

Solving the system of equations (6), (7) with consideration given to (12) and (13) makes it possible to determine the dependence of the degree of dissociation $\alpha$ (the conductance of the system is proportional to $\alpha$ ) on the relative humidity $P / P_{S}$.

The mechanisms of charge transfer are different at low and high humidity. At large values of $P / P_{S}$, virtually all of the channels are filled with water, and, therefore, ions move almost freely over these channels. At low values of $P / P_{S}$, many channels are blocked, and hydrated ions are forced to hop from one water-filled channel to another. Therefore, it is reasonable to divide the humidity scale into two ranges: $P / P_{S} \geq 0,3$ and $0,1<P / P_{S}<0,3$. To obtain analytical estimates at large humidity, we supposed that the dissociation energy is humidity-independent $\left(\partial E_{D} / \partial \beta \rightarrow 0, \beta>>1\right)$, a behavior typical of ionites with thin and relatively rigid $(U \sim 1)$ pores and channels and weakly dissociating IGs [15]. Thus, the solution to system of equation (6), (7) and (12), (13), takes the form

$$
P / P_{S} \approx \tilde{K}_{0}^{-1 / n} \alpha^{2 / n} \exp \left(U K_{0}^{-1 / n} \alpha^{2 / n}\right),
$$

where $K \square=\exp \left[-\left(E_{0}+E_{i d}\right) / T\right]=K_{0} \exp \left(-E_{i d} / T\right)$, and the dissociation constant is $K_{0}=\exp \left(-E_{0} / T\right)$.
In contrast, at low humidity, when the free volume of the pores is filled, the effect of electrostatic forces becomes predominant. Indeed, at $P / P_{S}<0,3$, the elastic deformation of the polymeric matrix is negligibly small, and dependence (14) takes the form

$$
\alpha \approx \tilde{K}_{0}^{1 / 2}\left(P / P_{S}\right)^{n / 2} .
$$

At the same time, a number of recent experimental studies have demonstrated a more substantial drop in the conductance over the indicated humidity range. It is intuitively clear that, at low humidity, where the contribution to the total thermodynamic potential from the elastic deformation energy is negligibly small, the main effect stems from the Coulomb forces associated with the surface charge and the spatial nonuniformity of the medium. In this case, the dependence of $E_{D}$ on the relative moisture content $\beta$ cannot be disregarded. Thus,

$$
\alpha \approx \tilde{K}_{0}^{1 / 2}\left(P / P_{S}\right)^{n / 2} \exp \left(-\frac{C_{1}}{P / P_{S}}\right),
$$

where $C_{1}=(C / 2) \ln \left[\left(\varepsilon+\varepsilon_{1}\right) /\left(2 \varepsilon_{1}\right)\right]$. In contrast to $(15)$, expression (16) suggests a sharper rise of the resistance at very low humidity.

To calculate the resistance of the system from the degree of dissociation $\alpha$, we assumed that the mobility of the ions $\mu$ is humidity-independent; i.e., the conductance $\lambda$ depends only on concentration $c$ of free carriers. Then the resistance of PI sample

$$
R=\frac{1}{\lambda} \frac{l}{S}=\frac{1}{\mu q c} \frac{l}{S},
$$

where $l$ is the characteristic length of conducting channel between electrodes and $S$ is the summary cross section area of all conducting channels.

Considered that the concentration of free ions in solution $c=\alpha N / S l$ we obtained

$$
R=\frac{l^{2}}{\mu q N} \frac{1}{\alpha}=\frac{R_{0}}{\alpha},
$$

where parameter $R_{0}=l^{2} /(\mu q N)$ depends on the geometry of the sample and the concentration of active IGs but does not depend on the humidity.

The empirical temperature dependence of the ion mobility $\mu$ can be presented as [16]

where $\gamma \approx 0,1$ and $t_{0}=15^{\circ} \mathrm{C}$.

$$
\mu=\mu_{0}\left(1+\gamma\left(t-t_{0}\right)\right) \text {, }
$$

\section{Theory and Experiment Comparison}

To obtain the experimental dependences of the resistance of PI sample on the relative air humidity, the special device was created. It consists of two-axis glass chamber with two lateral coaxial polishing cylinders. Three platinum coaxial electrodes are fixed on the each edge of these cylinders opposite one another. This device allows to measure the resistance of PI membrane by means of "pole" method, "double-membrane" method and diagonal method, and, consequently, to exclude the influence of contact resistances. Also device allows one to measure the mass and the thickness of samples in chamber directly. The measurements were performed with aid of alternating current bridge $(1000 \mathrm{~Hz})$. 
Figure 1 compares the measured and calculated dependences of the resistance of PI sample $R$ on the relative humidity $P / P_{S}$. The experiments on charge transfer were performed with lithium-form MF-4SK sulfocationite membranes with exchange capacity of 0,78 mg-equiv/g (sample mass is $20 \mathrm{mg}, N=9,36 \cdot 10^{18}$ ). The solid line represents the numerical solution to system of equations (6) and (7). The circles represent the experiment data. The theoretical dependences were calculated using the following values of the model parameters: $q=4,8 \cdot 10^{-10}$ esu, $\quad \varepsilon=79, \quad \varepsilon_{1}=4, \quad V_{W}=3 \cdot 10^{-23} \mathrm{~cm}^{3}$,

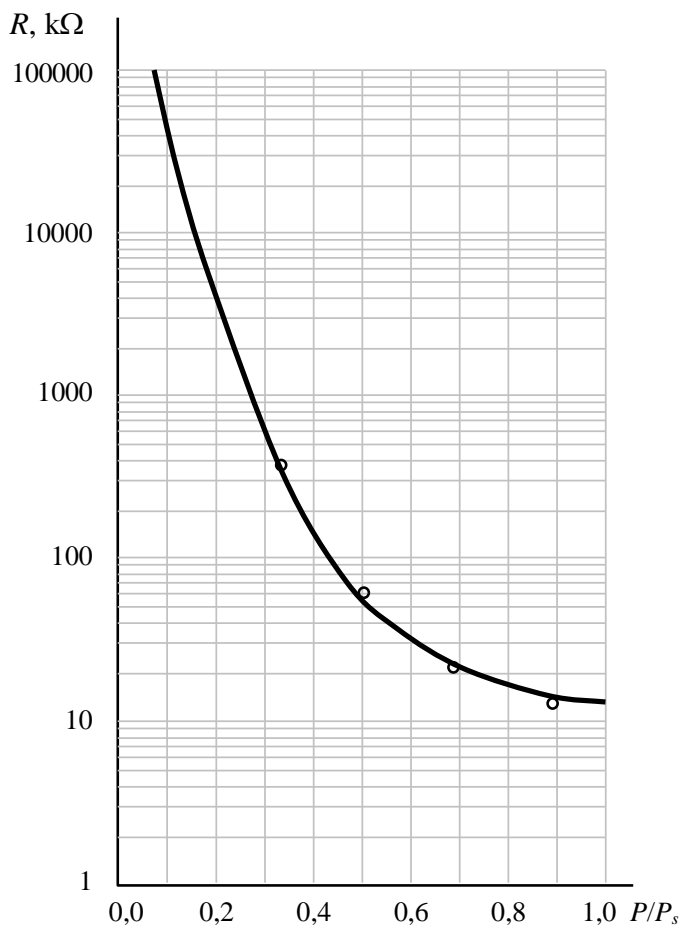

(a)

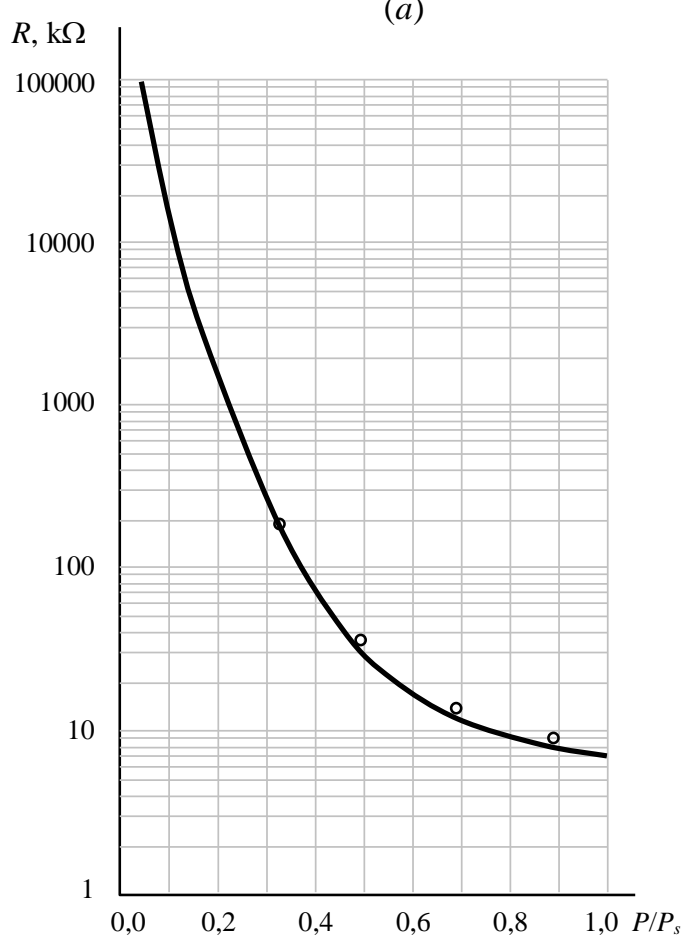

(c) $s=1,2 \cdot 10^{-15} \mathrm{~cm}^{2}, l=1 \mathrm{~cm}, n=4, \quad \beta_{0}=2$, and $U=1$; at $t=t_{0}=15^{\circ} \mathrm{C}, \quad \mu_{0}=3,2 \cdot 10^{-4} \mathrm{~cm}^{2} /(\mathrm{V} \cdot \mathrm{s}), \quad K_{0}=1,2$, and $R_{0}=2 \mathrm{k} \Omega$. The elastic deformation energy parameter $U$ was assumed to be temperature-independent.

Thus, the dependence of the resistance of the PI on the relative humidity predicted by the proposed model at the physically realistic parameters of the system is in close agreement with the corresponding experimental dependence at humidity from 10 to $100 \%$.

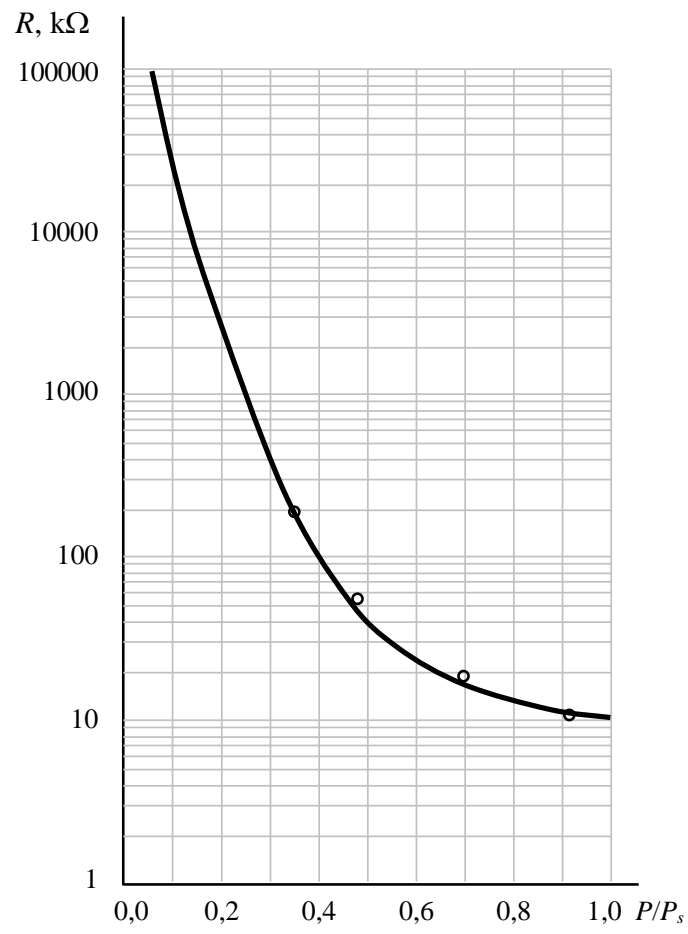

(b)

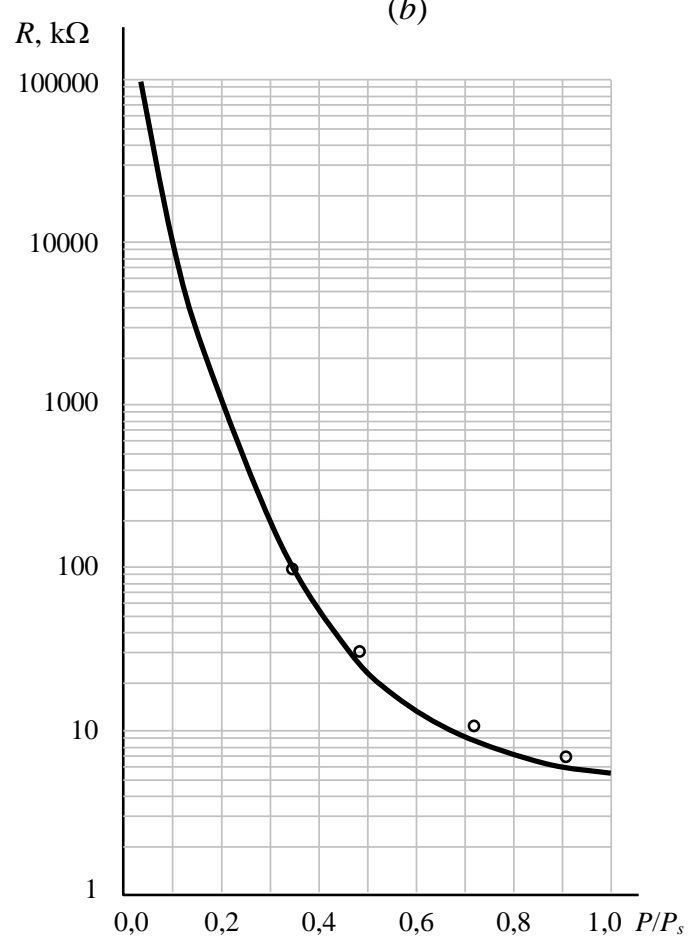

(d)

Fig. 1. The $R\left(P / P_{S}\right)$ dependence at various temperatures: (a) $15^{\circ} \mathrm{C} ;(b) 20^{\circ} \mathrm{C}$; (c) $30{ }^{\circ} \mathrm{C}$; (d) $40{ }^{\circ} \mathrm{C}$. 
At certain values of $C, \beta_{0}$ and $n$, the system can experience phase transitions that manifest themselves in a stepwise change in the degree of dissociation (and, hence, in the concentration of charge carriers and in resistance of PI) during variation of the humidity (see Fig. 2). The mechanisms and criteria of phase transitions of the thin adsorbed liquid film and thick adsorbed liquid film type were described in [4]. However, the analysis performed in the present work makes it possible to define more precisely these mechanisms and criteria and to relate them to the actual parameters of PI. The numerical analysis of (6) and (7) gives rise to the following criterion of phase transition $(n=4)$ :

$$
C \geq 4,6 \beta_{0},
$$

where numerical coefficient before $\beta_{0}$ is slightly decreased with $n$ reduction (if $n=1$ this coefficient equals 4,0), so that it can be set constant.

For example, such situation can appear in ionites with a small number of hydration of surface IGs $\left(\beta_{0}=1\right.$, Fig. 2, (2)) or with rarely placed IGs (large surface area $s$ per IG, Fig. 2, (3)). Therefore, it is necessary to take into account these facts in measurements of PI materials conductance. During the manufacturing of hygrometers, phase transitions should be avoided by selecting proper materials with noncritical parameters (Fig. 2, (1)).

According to [17], the water vapor adsorption hysteresis on sulfocationite membranes was discovered by some authors at high humidity $\left(0,6<P / P_{S}<0,8\right)$. Other authors discovered the hysteresis at low humidity $\left(P / P_{S} \leq 0,3\right)$. Note, these experimental results are in close agreement with the results of present work (as can be seen from Fig. 2, dependence 3 has hysteresis at high humidity and dependence 2 - at low). These authors explain the appearance of hysteresis as a result of capillary condensation. The present model explains the hysteresis by correlation between the water content and the dissociation of IGs: the fluctuating increase of water

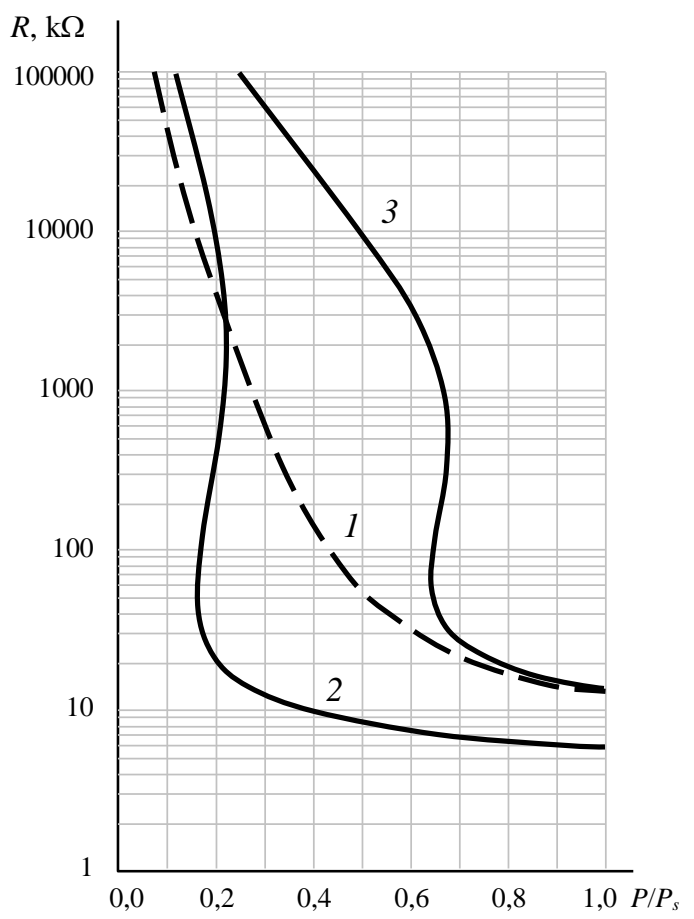

Fig. 2. The $R\left(P / P_{S}\right)$ dependences at $\beta_{0}=2, C=5,87$ (1);

$\beta_{0}=1, C=5,87$ (2); and $\beta_{0}=2, C=10,27$ (3). content leads to increase of IGs dissociation and, because of osmotic effect, the additional water molecules come into ionite. Moreover, this model explains the hysteresis at low humidity, when the capillary condensation is impossible in principle.

\section{Conclusion}

Thus, the results of the present work show that, when using the proposed model to interpret the available experimental data on the conduction of PIs in equilibrium with water vapor, it is fundamentally important to calculate exactly the electrostatic energy of ions in the sorbed water film.

Note, however, that for a further development of the mathematical model of charge transfer processes in porous PIs, it is necessary to verify experimentally the criterion (19) (i.e. to obtain the real values of polymer ionite parameters, which lead to a phase transitions) and to derive an analytical dependence of resistance of the ionite on the temperature. Such theoretical studies would allow one to formulate practical recommendations on selecting optimal polymeric coatings for the electrode units of molecular-electronic humidity gauges and to solve completely the problem of temperature correction of the response over as wide a range of humidity as possible.

This work was carried out using equipment provided by the Center of Collective Use of MSUT "STANKIN".

\section{References}

1. Y. Tanaka, Ion Exchange Membranes: Fundamentals and Applications (Elsevier, Tokyo, 2015).

2. L. Lazare, B. Sundheim, H. Gregor, J. Phys. Chem. 60, 641 (1956).

3. H. Gregor, B. Sundheim, K. Held, M. Waxman, J. Colloid Sci. 7, 511 (1952).

4. V.M. Agafonov, I.V. Ivanov, Russ. J. Phys. Chem. 70, 825 (1996).

5. V. Soldatov, V. Zelenkovskii, E. Kosandrovich, React. Funct. Polym. 102, 147 (2016).

6. I.V. Ivanov, Russ. J. Phys. Chem. 79, 448 (2005).

7. A.I. Gantman, Zh. Fiz. Khim. 66, 1713 (Rus) (1992).

8. N. Kononenko, V. Nikonenko, M. Fomenko, D. Grande, C. Larchet, L. Dammak, Y. Volfkovich, Adv. Colloid Interfac. 246, 196 (2017).

9. V.I. Vasil'eva, E.M. Akberova, V.A. Shaposhnik, M.D. Malykin, Russ. J. Electrochem. 50, 789 (2014).

10. Yu.A. Kokotov, Ion Exchangers and Ion Exchange (Khimiya, Leningrad, 1980).

11. S.F. Timashev, S.N. Cladkikh, Dokl. Akad. Nauk SSSR 262, 656 (1982).

12. L.D. Landau, E.M. Lifshits, Course of Theoretical Physics, Vol. 5: Statistical Physics, 2nd ed. (Nauka, Moscow, 1964; Pergamon, Oxford, 1980).

13. Ya.I. Frenkel', Kinetic Theory of Liquids (Nauka, Leningrad, 1975; Clarendon, Oxford, 1946).

14. V.P. Smilga, V.N. Gorelkin, in Investigation in the Field of Surface Forces (Nauka, Moscow, 1967), p. 174. 
15. Yu.M. Marichevskaya, O.D. Kurilenko, in Synthesis and Properties of Ion-Exchange Materials (Nauka, Moscow, 1968), p. 193.

16. L.I. Antropov, Theoretical Electrochemistry (Vysshaya Shkola, Moscow, 1975).

17. Yu.A. Kokotov, V.A. Pasechnik, Equilibrium and Kinetic of Ion Exchange (Khimiya, Leningrad, 1970). 